\title{
On the Generalized Krätzel Transform and Its Extension to Bohemian Spaces
}

\author{
S. K. Q. Al-Omari ${ }^{1}$ and Adem Kılıçman ${ }^{2}$ \\ ${ }^{1}$ Department of Applied Sciences, Faculty of Engineering Technology, Al-Balqa Applied University, Amman 11134, Jordan \\ ${ }^{2}$ Department of Mathematics and Institute of Mathematical Research, University of Putra Malaysia (UPM), 43400 Serdang, Selangor, \\ Malaysia
}

Correspondence should be addressed to Adem Kılıçman; akilic@upm.edu.my

Received 4 September 2013; Accepted 12 November 2013

Academic Editor: Mohammad Mursaleen

Copyright (c) 2013 S. K. Q. Al-Omari and A. Kılıçman. This is an open access article distributed under the Creative Commons Attribution License, which permits unrestricted use, distribution, and reproduction in any medium, provided the original work is properly cited.

We investigate the Krätzel transform on certain class of generalized functions. We propose operations that lead to the construction of desired spaces of generalized functions. The Krätzel transform is extended and some of its properties are obtained.

\section{Introduction}

In recent years, integral transforms of Bohemian have comprised an active area of research. Several integral transforms are extended to various spaces of Bohemian, especially, that permit a factorization property of Fourier convolution type.

On the other hand, several integral transforms that have not permitted a factorization property of Fourier convolution type are also extended to various spaces of Bohemian. In the sequence of these integral transforms, the Krätzel transform [1]

$$
\left(\mathscr{K}_{v}^{\rho} f\right)(x)=\int_{\mathbb{R}_{+}} \mathscr{Z}_{\rho}^{v}(x y) f(y) d y,
$$

where

$$
\mathscr{Z}_{\rho}^{v}(x y)=\int_{\mathbb{R}_{+}} t^{\nu-1} e^{-t^{\rho}-x y / t} d t,
$$

$\rho>0(\in \mathbb{N}), v \in \mathbb{C}, x>0$ is extended to certain space of ultra-Bohemian, denoted by $\mathcal{\delta}_{+}\left(l^{r}, \alpha,\left(\alpha_{i}\right), a\right)$ and $\mathcal{S}_{+}\left(l^{r}, \alpha,\left\{\alpha_{i}\right\}, a\right), 1 \leq r \leq \infty$, respectively.

Another form of the Krätzel transform was initially introduced in [2], and defined as a generalization of the Laplace transform in [3] as

$$
\left(l_{v}^{(\rho)} f\right)(x)=\int_{\mathbb{R}_{+}} \lambda_{v}^{(\rho)}(x y) f(y) d y,
$$

where

$$
\begin{aligned}
\lambda_{v}^{(\rho)}(x)= & \frac{(2 \pi)^{((\rho-1) / 2) \rho(1 / 2)}}{T(v+1-(1 / \rho))} \\
& \times\left(\frac{x}{\rho}\right)^{\rho v} \int_{1}^{\infty}\left(t^{\rho}-1\right)^{v-(1 / \rho)} e^{-x t} d t,
\end{aligned}
$$

for $\rho \in \mathbb{N}$ and $\operatorname{Re} v>-1+(1 / \rho), x>0$. The $l_{v}^{(\rho)}$ transform was extended to generalized functions in [3] and to distributions in $[4]$. By $l^{\rho}(X)$, we denote the space of equivalence classes of measurable functions $f: X \rightarrow \mathbb{R}$ such that

$$
\int_{X}|f|^{\rho} d \mu<\infty
$$

where two measurable functions are equivalent when they are equal to $\mu$ a.e.

For $l^{\rho}(X)$, the $l^{\rho}$-norm is defined as

$$
\|f\|_{l^{\rho}}=\left(\int_{X}|f|^{\rho} d \mu\right)^{1 / \rho} .
$$

The Banach space $l_{\rho, \mu}$ of Lebesgue measurable functions is defined by [3, page 446 ]

$$
\|f\|_{\rho, \mu}=\left\|x^{-\mu} f\right\|_{\rho}<\infty .
$$

Due to [3, Proposition 2.1], we state the following theorem. 
Theorem 1. Let $1 \leq \rho \leq \infty, \mu, v \in \mathbb{C}, \rho>0,(1 / \rho)+\left(1 / \rho^{\prime}\right)=$ 1 and $\operatorname{Re} \mu>-\left(1 / \rho^{\prime}\right)-\min \{0, \rho \operatorname{Re} v\}$ and then $l_{v}^{(\rho)}$ is a continuous linear mapping from $l_{\rho, \mu}$ into $l_{\rho, 2 /(\rho-\mu-1)}$.

Note that we always assume that the hypothesis of Theorem 1 is satisfied. By $\checkmark$ we denote the Mellin-type convolution product of first order defined by [5]

$$
(f \vee \varphi)(y)=\int_{\mathbb{R}_{+}} f\left(y t^{-1}\right) t^{-1} \varphi(t) d t .
$$

By $\mathscr{D}\left(\mathbb{R}_{+}\right)$, or simply $\mathscr{D}$, denote the Schwartz space of test functions of compact support defined on $\mathbb{R}_{+}$. Then we have the following definition.

Definition 2. Let $f, g \in l_{\rho, \mu}$. For $f$ and $g$, we define the operation $\otimes$ given by

$$
(f \otimes g)(x)=\int_{\mathbb{R}_{+}} f(x t) g(t) d t .
$$

Then we easily see that the operations in (8) and (9) are very basic for the next construction of the desired Bohemian spaces.

Theorem 3. Let $f \in l_{\rho, \mu}$ and $\varphi \in \mathscr{D}$, then $l_{v}^{(\rho)}(f \vee \varphi)(x)=$ $\left(\left(l_{v}^{(\rho)} f\right) \otimes \varphi\right)(x)$.

Proof. Let $x>0$. For $f \in l_{\rho, \mu}$ and $\varphi \in \mathscr{D}$ we, by (3) and (8), get that

$$
\begin{aligned}
l_{v}^{(\rho)} & (f \vee \varphi)(x) \\
& =\int_{\mathbb{R}_{+}}\left(\int_{\mathbb{R}_{+}} f\left(y t^{-1}\right) t^{-1} \varphi(t) d t\right) \lambda_{v}^{(\rho)}(x y) d y \\
& =\int_{\mathbb{R}_{+}}\left(\int_{\mathbb{R}_{+}} f\left(y t^{-1}\right) t^{-1} \varphi(t) d t\right) .
\end{aligned}
$$

A change of variables $y=t z$ in (10) implies

$$
\begin{aligned}
l_{v}^{(\rho)}(f \vee \varphi)(x) & =\int_{\mathbb{R}_{+}}\left(\int_{\mathbb{R}_{+}} f(z) \lambda_{v}^{(\rho)}(x t z) d z\right) \varphi(t) d t \\
& =\int_{\mathbb{R}_{+}}\left(l_{v}^{(\rho)} f\right)(x t) \varphi(t) d t .
\end{aligned}
$$

Hence, by (9), we have

$$
l_{v}^{(\rho)}(f \vee \varphi)(x)=\left(\left(l_{v}^{(\rho)} f\right) \otimes \varphi\right)(x) .
$$

This completes the proof of theorem.

\section{General Bohemian}

The structure necessary for the construction of Bohemian consists of the following:

(1) a group $X$ and a commutative semigroup $(Y, *)$;

(2) a operation $\odot: X \times Y \rightarrow X$ such that $x \odot\left(v_{1} * v_{2}\right)=$ $\left(x \odot v_{1}\right) \odot v_{2}$, for all $x \in X$ and $v_{1}, v_{2}, \in Y$;
(3) a collection $\Delta \subset Y^{\mathbb{N}}$ such that

(i) for all $\left(v_{n}\right),\left(\sigma_{n}\right) \in \Delta$, we have $\left(v_{n} * \sigma_{n}\right) \in \Delta$,

(ii) If $x \odot v_{n}=y \odot v_{n}$, then $x=y$ where $x, y \in$ $X,\left(v_{n}\right) \in \Delta$, and $n \in \mathbb{N}$.

Then the set $\Delta$ that satisfies (i) and (ii) is called the set of all delta sequences.

Let $A=\left\{\left(x_{n}, v_{n}\right): x_{n} \in X,\left(v_{n}\right) \in \Delta, x_{n} \odot v_{m}=x_{m} \odot\right.$ $v_{n}$, for all $\left.m, n \in \mathbb{N}\right\}$. Then we say $\left(x_{n}, v_{n}\right) \sim\left(y_{n}, \sigma_{n}\right)$ if there are $\left(x_{n}, v_{n}\right),\left(y_{n}, \sigma_{n}\right) \in A$, such that $x_{n} \odot \sigma_{m}=y_{m} \odot v_{n}$, for all $m, n \in \mathbb{N}$. The relation $\sim$ is an equivalence relation in $A$. The space of equivalence classes in $A$ is called the space of Bohemian and denoted by $\mathbb{B H}$. Each element of $\mathbb{B} \mathbb{H}$ is called Bohemian. Then the convergence in $\mathbb{B H}$ is defined as follows.

(1) $\left(h_{n}\right) \in \mathbb{B} \mathbb{H}$ is said to be $\delta$-convergent to $h \in \mathbb{B} \mathbb{H}$, denoted by $h_{n} \stackrel{\delta}{\rightarrow} h$ as $n \rightarrow \infty$, if $\exists$ a delta sequence $\left(v_{n}\right)$ such that $\left(h_{n} \odot v_{n}\right),\left(h \odot v_{n}\right) \in X$, and $\left(h_{n} \odot v_{k}\right) \rightarrow$ $\left(h \odot v_{k}\right) \in X$ as $n \rightarrow \infty$, for all $k, n \in \mathbb{N}$.

(2) $\left(h_{n}\right) \in \mathbb{B} \mathbb{W}$ is said to be $\Delta$-convergent to $h \in \mathbb{B} \mathbb{M}$, denoted by $h_{n} \stackrel{\Delta}{\rightarrow} h$ as $n \rightarrow \infty$, if $\exists$ a $\left(v_{n}\right) \in \Delta$ such that $\left(h_{n}-h\right) \odot v_{n} \in X$, for all $n \in \mathbb{N}$, and $\left(h_{n}-h\right) \odot v_{n} \rightarrow 0 \in X$ as $n \rightarrow \infty$.

The following theorem is equivalent to the statement of $\delta$ convergence.

Theorem 4. $h_{n} \stackrel{\delta}{\rightarrow} h \in \mathbb{B} \mathbb{W}$ as $n \rightarrow \infty$ if and only if there are $f_{n, k}, f_{k} \in X$ and $v_{k} \in \Delta$ such that $h_{n}=\left[f_{n, k} / v_{k}\right], h=\left[f_{k} / v_{k}\right]$ and, for all $k \in \mathbb{N}, f_{n, k} \rightarrow f_{k} \in X$ as $n \rightarrow \infty$. See [6-17] for more details.

\section{The Spaces $\mathbb{B H}_{1}\left(l_{\rho, \mu}\right)$ and $\mathbb{B H}_{2}\left(l_{\rho, 2 /(\rho-\mu-1)}\right)$}

In this section we construct the space $\mathbb{B H}\left(l_{\rho, \mu},(\mathscr{D}, \vee), \vee, \Delta\right)$ (or $\left.\mathbb{B H}_{1}\left(l_{\rho, \mu}\right)\right)$ and the space $\mathbb{B} \mathbb{H}\left(l_{\rho, 2 /(\rho-\mu-1)},(\mathscr{D}, \vee), \otimes, \Delta\right)$ (or $\left.\mathbb{B H}_{2}\left(l_{\rho, 2 /(\rho-\mu-1)}\right)\right)$ of Bohemian and give their properties.

At the first step, we prove the following connecting theorem.

Theorem 5. Let $f \in l_{\rho, \mu}$ and $\varphi, \psi \in \mathscr{D}$; then, $f \otimes(\varphi \vee \psi)=$ $(f \otimes \varphi) \otimes \psi$.

Proof. Let $f \in l_{\rho, \mu}$ and $\varphi, \psi \in \mathscr{D}$; then, applying the Fubinitz theorem yields

$$
\begin{aligned}
(f & \otimes(\varphi \vee \psi))(x) \\
& =\int_{\mathbb{R}_{+}} f(x t)(\varphi \vee \psi)(t) d t \\
& =\int_{\mathbb{R}_{+}} f(x t)\left(\int_{\mathbb{R}_{+}} \varphi\left(\frac{t}{y}\right) \psi(y) y^{-1} d y\right) d t \\
& =\int_{\mathbb{R}_{+}}\left(\int_{\mathbb{R}_{+}} f(x t) \varphi\left(\frac{t}{y}\right) y^{-1} d y\right) \psi(y) d y .
\end{aligned}
$$


The change of variables $t=y z$ implies $d t=y d z$ and further

$$
(f \otimes(\varphi \vee \psi))(x)=\int_{\mathbb{R}_{+}}\left(\int_{\mathbb{R}_{+}} f(x y z) \varphi(z) d z\right) \psi(y) d y .
$$

Therefore,

$$
(f \otimes(\varphi \vee \psi))(x)=\int_{\mathbb{R}_{+}}(f \otimes \varphi)(x y) \psi(y) d y .
$$

Hence, (15) implies

$$
(f \otimes(\varphi \vee \psi))(x)=((f \otimes \varphi) \otimes \psi)(x) .
$$

This completes the proof of the theorem.

Next, forthcoming theorems prove the existence of the space $\mathbb{B H}_{1}\left(l_{\rho, \mu}\right)$.

Theorem 6. Let $f \in l_{\rho, \mu}$ and $\varphi \in \mathscr{D}$ and then $f \vee \varphi \in l_{\rho, \mu}$.

Proof. For each $f \in l_{\rho, \mu}$ and $\varphi \in \mathscr{D}$, we have

$$
\left\|x^{\mu}(f \vee \varphi)(x)\right\|_{\rho, \mu}^{\rho}=\int_{\mathbb{R}_{+}}\left|x^{\mu} \int_{\mathbb{R}_{+}} f(x t) \varphi(t) d t\right|^{\rho} d x .
$$

By Jensen's theorem, we write

$$
\begin{aligned}
& \left\|x^{\mu}(f \vee \varphi)(x)\right\|_{\rho, \mu}^{\rho} \\
& \quad \leq \int_{\mathbb{R}_{+}} \int_{\mathbb{R}_{+}}\left|x^{\mu} \int_{\mathbb{R}_{+}} f(x t) \varphi(t) d t\right|^{\rho} d x \\
& \quad \leq \int_{\mathbb{R}_{+}} \int_{\mathbb{R}_{+}}\left|x^{\mu} f(x t)\right|^{\rho}|\varphi(t)| d t d x \\
& \quad \leq\|f\|_{\rho, \mu}^{\rho} \int_{K}|\varphi(t)| d t,
\end{aligned}
$$

where $K=[a, b], b>a>0$ is a compact subset containing the support of $\varphi$. Hence, from (18), we get

$$
\left\|x^{\mu}(f \vee \varphi)(x)\right\|_{\rho, \mu}^{\rho} \leq\|f\|_{\rho, \mu}^{\rho} M(b-a)
$$

where $M$ is certain positive real number. This completes the proof of the theorem.

Theorem 7. Let $f \in l_{\rho, \mu}$ and $\varphi, \psi \in \mathscr{D}$ and then

(i) $f \vee(\varphi+\psi)=f \vee \varphi+f \vee \psi$;

(ii) $(\alpha f) \vee \varphi=\alpha(f \vee \varphi)$;

(iii) let $\left(f_{n}\right) \in l_{\rho, \mu}$ and $f_{n} \rightarrow f$ as $n \rightarrow \infty$ then for $\varphi \in \mathscr{D}$, and we have $f_{n} \vee \varphi \rightarrow f \vee \varphi$ as $n \rightarrow \infty$;

(iv) $f \vee(\varphi \vee \psi)=(f \vee \varphi) \vee \psi$.

Proof of Part (i), (ii), and (iii) follows from properties of integral operators $\int$. Similarly, the proof of Part (iv) is straightforward from the properties of $\checkmark$ proved in [5].

Following theorem is straightforward, and detailed proof is omitted.
Theorem 8. Let $f \in l_{\rho, \mu}$ and $\left(\mu_{n}\right) \in \Delta$ then $f \vee \mu_{n} \rightarrow f$ as $n \rightarrow \infty$.

Proof. Since $\mathscr{D}$ is a dense subspace of $l_{\rho}$ then it is a dense subspace of $l_{\rho, \mu}$. Hence, there can be found $\psi \in \mathscr{D}$ such that

$$
\|f-\psi\|_{\rho, \mu}<\varepsilon
$$

for $\varepsilon>0$. Also, from (18), we have

$$
\left\|(f-\psi) \vee \mu_{n}\right\|_{\rho, \mu}^{\rho} \leq\|f\|_{\rho, \mu}^{\rho}\left\|\mu_{n}\right\|_{l^{1}} .
$$

Let $g(t)=y^{\mu} \psi\left(y t^{-1}\right) t^{-1}$ then; $g(t) \in \mathscr{D}$ and hence uniformly continuous on $\mathbb{R}_{+}$.

Therefore, there is $\delta>0$ such that

$$
|g(y)-g(x)|<\varepsilon \quad \text { whenever }|y-x|<\delta .
$$

Thus, using (22), we get

$$
\begin{aligned}
& \left\|\left(\psi \times \mu_{n}-\psi\right)(y)\right\|_{\rho, \mu}^{\rho} \\
& =\int_{\mathbb{R}_{+}}\left|y^{\mu}\left(\psi \times \mu_{n}-\psi\right)(y)\right|^{\rho} d y \\
& \leq \int_{\mathbb{R}_{+}} \int_{\mathbb{R}_{+}} \mid y^{\mu}\left(\psi\left(y t^{-1}\right) t^{-1} \mu_{n}(t)\right. \\
& \leq \int_{\mathbb{R}_{+}} \int_{\mathbb{R}_{+}}\left|y^{\mu}\left(\psi\left(y t^{-1}\right) t^{-1}-\psi(y)\right)\right|^{\rho} \\
& =\int_{\mathbb{R}_{+}} \int_{\mathbb{R}_{+}}|g(y)-g(1)|^{\rho}\left|\mu_{n}(t)\right| d t d y \\
& =\int_{\mathbb{R}_{+}} \int_{\mathbb{R}_{+}} \varepsilon^{\rho}\left|\mu_{n}(t)\right| d t d y .
\end{aligned}
$$

By (22), supp $\mu_{n}(t) \rightarrow 0$ as $n \rightarrow \infty$ implies that there can be found $N \in \mathbb{N}$, such that supp $\mu_{n} \subseteq[0, \delta]$, for all $n \geq N$. Further, the fact that the function $\psi$ is of compact support thus this implies that $\operatorname{supp} \psi(y) \subseteq K=[a, b]$, where $K$ is a compact subset of $\mathbb{R}_{+}$. Thus, from (23), we write

$$
\begin{aligned}
\left\|\psi \vee \mu_{n}-\psi\right\|_{\rho, \mu}^{\rho} & \leq \varepsilon^{\rho} \int_{a}^{b} \int_{0}^{\delta} M d t \\
& =\varepsilon^{\rho}(b-a)(2 \delta) M .
\end{aligned}
$$

Now, we have

$$
\begin{aligned}
\left\|f \times \mu_{n}-f\right\|_{\rho, \mu} \leq & \left\|(f-\psi) \vee \mu_{n}\right\|_{\rho, \mu} \\
& +\left\|\psi \times \mu_{n}-\psi\right\|_{\rho, \mu}+\|(f-\psi)\|_{\rho, \mu} .
\end{aligned}
$$

On using (20), (21) and (24) prove that

$$
\left\|f \times \mu_{n}-f\right\|_{\rho, \mu} \longrightarrow 0 \quad \text { as } \epsilon \longrightarrow 0 .
$$


Thus the theorem is proved. Then the Bohemian space $\mathbb{B H}_{1}\left(l_{\rho, \mu}\right)$ is therefore constructed. The operations such as sum and multiplication by a scalar of two Bohemian in $\mathbb{B H}_{1}\left(l_{\rho, \mu}\right)$ are defined in a natural way

$$
\begin{gathered}
{\left[\frac{\left(f_{n}\right)}{\left(\mu_{n}\right)}\right]+\left[\frac{\left(g_{n}\right)}{\left(\tau_{n}\right)}\right]=\left[\frac{\left(f_{n} \vee \tau_{n}+g_{n} \vee \mu_{n}\right)}{\left(\mu_{n} \vee \tau_{n}\right)}\right],} \\
\alpha\left[\frac{\left(f_{n}\right)}{\left(\mu_{n}\right)}\right]=\left[\frac{\left(a f_{n}\right)}{\left(\mu_{n}\right)}\right],
\end{gathered}
$$

where $\alpha$ is a complex number. by

Similarly, the operation $\vee$ and differentiation are defined

$$
\begin{gathered}
{\left[\frac{\left(f_{n}\right)}{\left(\mu_{n}\right)}\right] \vee\left[\frac{\left(g_{n}\right)}{\left(\tau_{n}\right)}\right]=\left[\frac{\left(f_{n} \vee g_{n}\right)}{\left(\mu_{n} \vee \tau_{n}\right)}\right],} \\
\mathscr{D}^{\alpha}\left[\frac{\left(f_{n}\right)}{\left(\mu_{n}\right)}\right]=\left[\frac{\left(\mathscr{D}^{\alpha} f_{n}\right)}{\left(\mu_{n}\right)}\right] .
\end{gathered}
$$

Now constructing the space $\mathbb{B H}_{2}\left(l_{\rho, 2 /(\rho-\mu-1)}\right)$ follows from theorems which were used for constructing the space $\mathbb{B H}_{1}\left(l_{\rho, \mu}\right)$. Therefore, the corresponding proofs of Theorems 10 and 11 are omitted.

Theorem 9. Let $f \in l_{\rho, 2 /(\rho-\mu-1)}$ and $\varphi \in \mathscr{D}$ and then $f \otimes \varphi \in$ $l_{\rho, 2 /(\rho-\mu-1)}$.

Theorem 10. Let $f \in l_{\rho, 2 /(\rho-\mu-1)}, \varphi, \psi \in \mathscr{D}$ and then the following hold:

(i) $f \otimes(\varphi+\psi)=f \otimes \varphi+f \otimes \psi$;

(ii) $(\alpha f) \otimes \varphi=\alpha(f \otimes \varphi)$;

(iii) if $f_{n} \rightarrow f$ in $l_{\rho, 2 /(\rho-\mu-1)}$, as $n \rightarrow \infty$ then $f_{n} \otimes \varphi \rightarrow$ $f \otimes \varphi$ as $n \rightarrow \infty$;

(iv) if $\left(\mu_{n}\right) \in \Delta$, then $f \otimes \mu_{n} \rightarrow f$ as $n \rightarrow \infty$.

Theorem 11. Let $f \in l_{\rho, 2 /(\rho-\mu-1)}$ and $\varphi, \psi \in \mathscr{D}$ and then $f \otimes$ $(\varphi \vee \psi)=(f \otimes \varphi) \otimes \psi$.

Proof of this theorem is similar to that of Theorem 6. Thus the space $\mathbb{B} \mathbb{H}_{2}\left(l_{\rho, 2 /(\rho-\mu-1)}\right)$ can be regarded as Bohemian space.

\section{Krätzel Transform of Bohemian}

By aid of Theorem 4, we have the right to define the Krätzel transform of $\left[\left(f_{n}\right) /\left(\mu_{n}\right)\right] \in \mathbb{B H}_{1}\left(l_{\rho, \mu}\right)$ as the Bohemian

$$
\widehat{l_{v}^{(\rho)}}\left[\frac{\left(f_{n}\right)}{\left(\mu_{n}\right)}\right]=\left[\frac{\left(l_{v}^{(\rho)} f_{n}\right)}{\left(\mu_{n}\right)}\right]
$$

is embedded in the space $\mathbb{B H}_{2}\left(l_{\rho, 2 /(\rho-\mu-1)}\right)$.

Theorem 12. The mapping $\widehat{l_{v}^{(\rho)}}: \mathbb{B H}_{1}\left(l_{\rho, \mu}\right) \quad \rightarrow$ $\mathbb{B H}_{2}\left(l_{\rho, 2 /(\rho-\mu-1)}\right)$ is

(i) well defined,

(ii) linear.
Proof. Let $\left[\left(f_{n}\right) /\left(\mu_{n}\right)\right],\left[\left(g_{n}\right) /\left(\psi_{n}\right)\right] \in \mathbb{B H}_{1}\left(l_{\rho, \mu}\right)$ be such that $\left[\left(f_{n}\right) /\left(\mu_{n}\right)\right]=\left[\left(g_{n}\right) /\left(\psi_{n}\right)\right]$, and then $f_{n} \vee \psi_{m}=g_{m} \vee \mu_{n}=$ $g_{n} \vee \mu_{m}$. Using Theorem 4 implies $l_{v}^{(\rho)} f_{n} \otimes \psi_{m}=l_{v}^{(\rho)} g_{n} \otimes \mu_{m}$, for all $n, m$. The idea of quotient of sequences in $\mathbb{B H}_{2}\left(l_{\rho, 2 /(\rho-\mu-1)}\right)$ implies that

$$
\frac{l_{v}^{(\rho)} f_{n}}{\mu_{n}} \text { is equivalent to } \frac{l_{v}^{(\rho)} g_{n}}{\psi_{n}}
$$

That is,

$$
\left[\frac{\left(l_{v}^{(\rho)} f_{n}\right)}{\left(\mu_{n}\right)}\right]=\left[\frac{\left(l_{v}^{(\rho)} g_{n}\right)}{\left(\psi_{n}\right)}\right]
$$

To prove part (ii) of the theorem, if $\left[\left(f_{n}\right) /\left(\mu_{n}\right)\right],\left[\left(g_{n}\right) /\left(\psi_{n}\right)\right] \epsilon$ $\mathbb{B H}_{1}\left(l_{\rho, \mu}\right)$, then

$$
\widehat{l_{v}^{(\rho)}}\left(\left[\frac{\left(f_{n}\right)}{\left(\mu_{n}\right)}\right]+\left[\frac{\left(g_{n}\right)}{\left(\psi_{n}\right)}\right]\right)=\left[\frac{\left(l_{v}^{(\rho)} f_{n} \otimes \psi_{n}+l_{v}^{(\rho)} g_{n} \otimes \mu_{n}\right)}{\left(\mu_{n} \otimes \psi_{n}\right)}\right] .
$$

Hence

$$
\widehat{l_{v}^{(\rho)}}\left(\left[\frac{\left(f_{n}\right)}{\left(\mu_{n}\right)}\right]+\left[\frac{\left(g_{n}\right)}{\left(\psi_{n}\right)}\right]\right)=\widehat{l_{v}^{(\rho)}}\left[\frac{\left(f_{n}\right)}{\left(\mu_{n}\right)}\right]+\widehat{l_{v}^{(\rho)}}\left[\frac{\left(g_{n}\right)}{\left(\psi_{n}\right)}\right] .
$$

Also, if $\alpha \in \mathbb{C}$, then

$$
\alpha l_{v}^{(\rho)}\left[\frac{\left(f_{n}\right)}{\left(\mu_{n}\right)}\right]=\alpha\left[\frac{\left(l_{v}^{(\rho)} f_{n}\right)}{\left(\mu_{n}\right)}\right]=\left[\frac{\left(l_{v}^{(\rho)}\left(\alpha f_{n}\right)\right)}{\left(\mu_{n}\right)}\right] .
$$

Hence

$$
\alpha \widehat{l_{v}^{(\rho)}}\left[\frac{\left(f_{n}\right)}{\left(\mu_{n}\right)}\right]=\widehat{l_{v}^{(\rho)}}\left(\alpha\left[\frac{\left(f_{n}\right)}{\left(\mu_{n}\right)}\right]\right) .
$$

This completes the proof.

Definition 13. Let $\left[\left(l_{v}^{(\rho)} f_{n}\right) /\left(\mu_{n}\right)\right] \in \mathbb{B H}_{2}\left(l_{\rho, 2 /(\rho-\mu-1)}\right)$. We define the inverse of $\widehat{l_{v}^{(\rho)}}$ transform of the Bohemian $\left[\left(l_{v}^{(\rho)} f_{n}\right) /\left(\mu_{n}\right)\right]$ as

$$
\widehat{l}_{v}^{(\rho)}\left[\frac{\left(l_{v}^{(\rho)} f_{n}\right)}{\left(\mu_{n}\right)}\right]=\left[\frac{\left(l_{v}^{(\rho)}\right)^{-1}\left(l_{v}^{(\rho)} f_{n}\right)}{\left(\mu_{n}\right)}\right]=\left[\frac{\left(f_{n}\right)}{\left(\mu_{n}\right)}\right],
$$

for each $\left(\mu_{n}\right) \in \Delta$.

Theorem 14. $\widehat{l_{v}^{(\rho)}}: \mathbb{B H}_{1}\left(l_{\rho, \mu}\right) \rightarrow \mathbb{B H}_{2}\left(l_{\rho, 2 /(\rho-\mu-1)}\right)$ is an isomorphism.

Proof. Let $\widehat{l_{v}^{(\rho)}}\left[\left(f_{n}\right) /\left(\mu_{n}\right)\right]=\widehat{l_{v}^{(\rho)}}\left[\left(g_{n}\right) /\left(\psi_{n}\right)\right] \in \mathbb{B} \mathbb{H}_{2}\left(l_{\rho, 2 /(\rho-\mu-1)}\right)$ and then by (29) we get $l_{v}^{(\rho)} f_{n} \otimes \psi_{m}=l_{v}^{(\rho)} g_{m} \otimes \mu_{n}$. Therefore, Theorem 4 implies

$$
l_{v}^{(\rho)}\left(f_{n} \vee \psi_{m}\right)=l_{v}^{(\rho)}\left(g_{m} \vee \mu_{n}\right) .
$$


Thus $f_{n} \vee \psi_{m}=g_{m} \vee \mu_{n}$, for all $m, n \in \mathbb{N}$. The concept of quotients of equivalent classes of $\mathbb{B} \mathbb{H}_{1}\left(l_{\rho, \mu}\right)$ then gives

$$
\left[\frac{\left(f_{n}\right)}{\left(\mu_{n}\right)}\right]=\left[\frac{\left(g_{n}\right)}{\left(\psi_{n}\right)}\right] .
$$

This proves that $\widehat{l_{v}^{(\rho)}}$ is injective.

To show that $l_{v}^{(\rho)}$ is surjective, let $\left[\left(l_{v}^{(\rho)} f_{n}\right) /\left(\mu_{n}\right)\right] \in$ $\mathbb{B H}_{2}\left(l_{\rho, 2 /(\rho-\mu-1)}\right)$. Then $\left(l_{v}^{(\rho)} f_{n}\right) /\left(\mu_{n}\right)$ is a quotient of sequences in $\mathbb{B H}_{2}\left(l_{\rho, 2 /(\rho-\mu-1)}\right)$. Hence, $l_{v}^{(\rho)} f_{n} \otimes \mu_{m}=l_{v}^{(\rho)} f_{m} \otimes \mu_{n}$, for all $m, n \in \mathbb{N}$. Once again, Theorem 4 implies that $l_{v}^{(\rho)}\left(f_{n} \vee \mu_{m}\right)=$ $l_{v}^{(\rho)}\left(f_{m} \vee \mu_{n}\right)$. Hence $\left[\left(f_{n}\right) /\left(\mu_{n}\right)\right] \in \mathbb{B H}_{1}\left(l_{\rho, \mu}\right)$ satisfies

$$
l_{v}^{(\rho)}\left[\frac{\left(f_{n}\right)}{\left(\mu_{n}\right)}\right]=\left[\frac{\left(l_{v}^{(\rho)} f_{n}\right)}{\left(\mu_{n}\right)}\right]
$$

This completes the proof of the theorem.

Theorem 15. Let $\psi \in \quad \mathscr{D}$ and $\left[\left(l_{v}^{(\rho)} f_{n}\right) /\left(\mu_{n}\right)\right] \quad \epsilon$ $\mathbb{B H}_{2}\left(l_{\rho, 2 /(\rho-\mu-1)}\right)$ then one has
(i) ${\widehat{l_{v}^{(\rho)}}}^{-1}\left(\left[\left(l_{v}^{(\rho)} f_{n}\right) /\left(\mu_{n}\right)\right] \otimes \psi\right)=\left[\left(f_{n}\right) /\left(\mu_{n}\right)\right] \vee \psi$;
(ii) $\widehat{l_{v}^{(\rho)}}\left(\left[\left(f_{n}\right) /\left(\mu_{n}\right)\right] \vee \psi\right)=\left[\left(l_{v}^{(\rho)} f_{n}\right) /\left(\mu_{n}\right)\right] \otimes \psi$.

Proof. By using (29), we have

$$
{\widehat{l_{v}^{(\rho)}}}^{-1}\left(\left[\frac{\left(l_{v}^{(\rho)} f_{n}\right)}{\left(\mu_{n}\right)}\right] \otimes \psi\right)=\left[\frac{\left(l_{v}^{(\rho)}\right)^{-1}\left(\left(l_{v}^{(\rho)} f_{n}\right) \otimes \psi\right)}{\left(\mu_{n}\right)}\right] .
$$

Theorem 4 then gives

$$
{\widehat{l_{v}^{(\rho)}}}^{-1}\left(\left[\frac{\left(l_{v}^{(\rho)} f_{n}\right)}{\left(\mu_{n}\right)}\right] \otimes \psi\right)=\left[\frac{\left(f_{n}\right) \vee \psi}{\left(\mu_{n}\right)}\right]=\left[\frac{\left(f_{n}\right)}{\left(\mu_{n}\right)}\right] \vee \psi .
$$

Hence the part (i) of the theorem is proved. The proof of part (ii) is similar thus we omit the details. This completes the proof of the theorem.

\section{Theorem 16. The mappings}

(i) $\widehat{l_{v}^{(\rho)}}: \mathbb{B H}_{1}\left(l_{\rho, \mu}\right) \rightarrow \mathbb{B H}_{2}\left(l_{\rho, 2 /(\rho-\mu-1)}\right)$ are continuous with respect to $\delta$ and $\Delta$-convergence.

(ii) $\widehat{l}_{v}^{(\rho)}: \mathbb{B H}_{2}\left(l_{\rho, 2 /(\rho-\mu-1)}\right) \rightarrow \mathbb{B H}_{1}\left(l_{\rho, \mu}\right)$ are continuous with respect to $\delta$ and $\Delta$-convergence.

Proof. At first, let us show that $\widehat{l}_{v}^{(\rho)}$ and ${\widehat{l_{v}^{(\rho)}}}^{-1}$ are continuous with respect to $\delta$-convergence.

Let $\beta_{n} \stackrel{\delta}{\rightarrow} \beta$ in $\mathbb{B H}_{1}\left(l_{\rho, \mu}\right)$ as $n \rightarrow \infty$ and then we show that $\widehat{l_{v}^{(\rho)}} \beta_{n} \rightarrow \widehat{l_{v}^{(\rho)}} \beta$ as $n \rightarrow \infty$. By virtue of Theorem 5, we can find $f_{n, k}$ and $f_{k}$ in $l_{\rho, \mu}$ such that

$$
\beta_{n}=\left[\frac{f_{n, k}}{\mu_{k}}\right], \quad \beta=\left[\frac{f_{k}}{\mu_{k}}\right]
$$

such that $f_{n, k} \rightarrow f_{k}$ as $n \rightarrow \infty$ for every $k \in \mathbb{N}$. Hence, $l_{v}^{(\rho)} f_{n, k} \rightarrow l_{v}^{(\rho)} f_{k} \in l_{\rho, 2 /(\rho-\mu-1)}$ as $n \rightarrow \infty$. Thus,

$$
\left[\frac{l_{v}^{(\rho)} f_{n, k}}{\mu_{k}}\right] \longrightarrow\left[\frac{l_{v}^{(\rho)} f_{k}}{\mu_{k}}\right] \in \mathbb{B H}_{2}\left(l_{\rho, 2 /(\rho-\mu-1)}\right)
$$

as $n \rightarrow \infty$.

To prove the Part (ii), Let $g_{n}, g \in \mathbb{B H}_{2}\left(l_{\rho, 2 /(\rho-\mu-1)}\right)$ be such that $g_{n} \stackrel{\delta}{\rightarrow} g$ as $n \rightarrow \infty$. Then, once again, by Theorem 5 , $g_{n}=\left[l_{v}^{(\rho)} f_{n, k} / \mu_{k}\right]$ and $g=\left[l_{v}^{(\rho)} f_{k} / \mu_{k}\right]$ for some $f_{n, k}, f_{k} \in$ $l_{\rho, \mu}$ and $l_{v}^{(\rho)} f_{n, k} \rightarrow l_{v}^{(\rho)} f_{k}$ as $n \rightarrow \infty$. Hence $\left[f_{n, k} / \mu_{k}\right] \rightarrow$ $\left[f_{k} / \mu_{k}\right]$ as $n \rightarrow \infty$.

Using (36), we get

$$
{\widehat{l_{v}^{(\rho)}}}^{-1}\left[\frac{l_{v}^{(\rho)} f_{n, k}}{\mu_{k}}\right] \longrightarrow \widehat{l}_{v}^{(\rho)}{ }^{-1}\left[\frac{l_{v}^{(\rho)} f_{k}}{\mu_{k}}\right] \quad \text { as } n \rightarrow \infty .
$$

Now, we establish that $\widehat{l}_{v}^{(\rho)}$ and ${\widehat{l_{v}^{(\rho)}}}^{-1}$ are continuous with respect to $\Delta$-convergence.

Let $\beta_{n} \stackrel{\Delta}{\rightarrow} \beta$ in $\mathbb{B H}_{1}\left(l_{\rho, \mu}\right)$ as $n \rightarrow \infty$. Then, there exist $f_{n} \in l_{\rho, \mu}$ and $\left(\mu_{n}\right) \in \Delta$ such that $\left(\beta_{n}-\beta\right) \vee \mu_{n}=\left[\left(\left(f_{n}\right) \vee \mu_{k}\right) / \mu_{k}\right]$ and $f_{n} \rightarrow 0$ as $n \rightarrow \infty$. By applying (29) we get

$$
\widehat{l_{v}^{(\rho)}}\left(\left(\beta_{n}-\beta\right) \vee \mu_{n}\right)=\left[\frac{l_{v}^{(\rho)}\left(\left(f_{n}\right) \vee \mu_{k}\right)}{\mu_{k}}\right] \text {. }
$$

Hence, we have $\widehat{l_{v}^{(\rho)}}\left(\left(\beta_{n}-\beta\right) \vee \mu_{n}\right)=\left[\left(\left(l_{v}^{(\rho)} f_{n}\right) \otimes \mu_{k}\right) / \mu_{k}\right]=$ $l_{v}^{(\rho)} f_{n} \rightarrow 0$ as $n \rightarrow \infty$ in $l_{\rho, 2 /(\rho-\mu-1)}$.

Therefore

$$
\widehat{l_{v}^{(\rho)}}\left(\left(\beta_{n}-\beta\right) \vee \mu_{n}\right)=\left(\widehat{l_{v}^{(\rho)}} \beta_{n}-\widehat{l_{v}^{(\rho)}} \beta\right) \otimes \mu_{n} \longrightarrow 0
$$

$$
\text { as } n \longrightarrow \infty \text {. }
$$

Hence, $\widehat{l_{v}^{(\rho)}} \beta_{n} \stackrel{\Delta}{\rightarrow} \widehat{l_{v}^{(\rho)}} \beta$ as $n \rightarrow \infty$.

Finally, let $g_{n} \stackrel{\Delta}{\rightarrow} g \in \mathbb{B H}_{2}\left(l_{\rho, 2 /(\rho-\mu-1)}\right)$ as $n \rightarrow \infty$ and then we find $l_{v}^{(\rho)} f_{k} \in l_{\rho, 2 /(\rho-\mu-1)}$ such that $\left(g_{n}-g\right) \otimes \mu_{n}=$ $\left[\left(l_{v}^{(\rho)} f_{k} \otimes \mu_{k}\right) / \mu_{k}\right]$ and $l_{v}^{(\rho)} f_{k} \rightarrow 0$ as $n \rightarrow \infty$ for some $\left(\mu_{n}\right) \epsilon$ $\Delta$.

Now, using (36), we obtain

$$
{\widehat{l_{v}^{(\rho)}}}^{-1}\left(\left(g_{n}-g\right) \otimes \mu_{n}\right)=\left[\frac{\left(l_{v}^{(\rho)}\right)^{-1}\left(l_{v}^{(\rho)} f_{k} \otimes \mu_{k}\right)}{\mu_{k}}\right] \text {. }
$$

Theorem 4 implies

$$
\begin{aligned}
\widehat{l}_{v}^{(\rho)}\left(\left(g_{n}-g\right) \otimes \mu_{n}\right)=\left[\frac{\left(f_{n}\right) \vee \mu_{k}}{\mu_{k}}\right] & =f_{n} \longrightarrow 0 \\
\text { as } n & \longrightarrow \infty \text { in } l_{\rho, \mu} .
\end{aligned}
$$

Thus

$$
\begin{aligned}
& \widehat{l}_{v}^{(\rho)}\left(\left(g_{n}-g\right) \otimes \mu_{n}\right) \\
& =\left(\widehat{l}_{v}^{(\rho)}{ }^{-1} g_{n}-\widehat{l}_{v}^{(\rho)}-1\right.
\end{aligned}
$$


From this, we find that ${\widehat{l_{v}^{(\rho)}}}^{-1} g_{n} \stackrel{\Delta}{\rightarrow}{\widehat{l_{v}^{(\rho)}}}^{-1} g$ as $n \rightarrow \infty$ in $\mathbb{B} \mathbb{T}_{1}\left(l_{\rho, \mu}\right)$.

This completes the proof of the theorem.

Theorem 17. The $\widehat{l_{v}^{(\rho)}}$ transform is consistent with the classical transform $l_{v}^{(\rho)}$.

Proof. For every $f \in l_{\rho, \mu}$, let $\beta$ be its representative in $\mathbb{B H}_{1}\left(l_{\rho, \mu}\right)$ and then $\beta=\left[\left(f \vee\left(\mu_{n}\right)\right) /\left(\mu_{n}\right)\right]$ where $\left(\mu_{n}\right) \in \Delta$, for all $n \in \mathbb{N}$. Since $\left(\mu_{n}\right)$ is independent from the representative, for all $n \in \mathbb{N}$, therefore

$$
\widehat{l_{v}^{(\rho)}}(\beta)=\widehat{l_{v}^{(\rho)}}\left(\left[\frac{f \vee\left(\mu_{n}\right)}{\left(\mu_{n}\right)}\right]\right)=\left[\frac{l_{v}^{(\rho)} f \otimes\left(\mu_{n}\right)}{\left(\mu_{n}\right)}\right],
$$

which is the representative of $l_{v}^{(\rho)} f \in l_{\rho, 2 /(\rho-\mu-1)}$. Thus this completes the proof.

Theorem 18. Let $\left[\left(g_{n}\right) /\left(\psi_{n}\right)\right] \in \mathbb{B H}_{2}\left(l_{\rho, 2 /(\rho-\mu-1)}\right)$ and then $a$ necessary and sufficient condition for $\left[\left(g_{n}\right) /\left(\psi_{n}\right)\right]$ to be in the range of $l_{v}^{\widehat{(\rho)}}$ is that $g_{n}$ belongs to range of $l_{v}^{(\rho)}$, for all $n \in \mathbb{N}$.

Proof. Let $\left[\left(g_{n}\right) /\left(\psi_{n}\right)\right]$ be in the range of $\widehat{l_{v}^{(\rho)}}$ and then it is clear that $g_{n}$ belongs to the range of $l_{v}^{(\rho)}$, for all $n \in \mathbb{N}$.

To establish the converse, let $g_{n}$ be in the range of $l_{v}^{(\rho)}$, for all $n \in \mathbb{N}$. Then there is $f_{n} \in l_{\rho, \mu}$ such that $l_{v}^{(\rho)} f_{n}=g_{n}, n \in \mathbb{N}$. Since $\left[\left(g_{n}\right) /\left(\psi_{n}\right)\right] \in \mathbb{B H}_{2}\left(l_{\rho, 2 /(\rho-\mu-1)}\right)$,

$$
g_{n} \otimes \psi_{m}=g_{m} \otimes \psi_{n}
$$

for all $m, n \in \mathbb{N}$, therefore,

$$
l_{v}^{(\rho)}\left(f_{n} \vee \varphi_{n}\right)=l_{v}^{(\rho)}\left(f_{m} \vee \varphi_{n}\right), \quad \forall m, n \in \mathbb{N},
$$

where $f_{n} \in l_{\rho, \mu}$ and $\varphi_{n} \in \Delta$, for all $n \in \mathbb{N}$. Then it follows that we get $f_{n} \vee \varphi_{m}=f_{m} \vee \varphi_{n}$, for all $m, n \in \mathbb{N}$. Thus $f_{n} / \varphi_{n}$ is a quotient of sequences in $\mathbb{B H}_{1}\left(l_{\rho, \mu}\right)$. Therefore, we have $\left[\left(f_{n}\right) /\left(\varphi_{n}\right)\right] \in \mathbb{B H}_{1}\left(l_{\rho, \mu}\right)$ and

$$
\widehat{l_{v}^{(\rho)}}\left(\left[\frac{\left(f_{n}\right)}{\left(\varphi_{n}\right)}\right]\right)=\left[\frac{\left(g_{n}\right)}{\left(\psi_{n}\right)}\right] .
$$

Hence the theorem is proved.

Theorem 19. If $\beta=\left[\left(f_{n}\right) /\left(\varphi_{n}\right)\right] \in \mathbb{B H}_{1}\left(l_{\rho, \mu}\right)$ and $\gamma=$ $\left[\left(\kappa_{n}\right) /\left(\mu_{n}\right)\right] \in \mathbb{B H}_{1}\left(l_{\rho, \mu}\right)$, then one has

$$
\widehat{l_{v}^{(\rho)}}\left(\left[\frac{\left(f_{n}\right)}{\left(\varphi_{n}\right)}\right] \vee\left[\frac{\left(\kappa_{n}\right)}{\left(\mu_{n}\right)}\right]\right)=\left[\frac{\left(l_{v}^{(\rho)} f_{n}\right)}{\left(\varphi_{n}\right)}\right] \otimes\left[\frac{\left(\kappa_{n}\right)}{\left(\mu_{n}\right)}\right]
$$

Proof. Let the hypothesis of the theorem be satisfied for some $\left[\left(f_{n}\right) /\left(\varphi_{n}\right)\right]$ and $\left[\left(\kappa_{n}\right) /\left(\mu_{n}\right)\right]$. Therefore,

$$
\begin{aligned}
\widehat{l_{v}^{(\rho)}}\left(\left[\frac{\left(f_{n}\right)}{\left(\varphi_{n}\right)}\right] \vee\left[\frac{\left(\kappa_{n}\right)}{\left(\mu_{n}\right)}\right]\right) & =\widehat{l_{v}^{(\rho)}}\left(\left[\frac{\left(f_{n} \vee \kappa_{n}\right)}{\left(\varphi_{n} \vee \mu_{n}\right)}\right]\right) \\
& =\left[\frac{\left(\left(l_{v}^{(\rho)} f_{n}\right) \otimes \kappa_{n}\right)}{\left(\varphi_{n} \otimes \mu_{n}\right)}\right] \\
& =\left[\frac{\left(l_{v}^{(\rho)} f_{n}\right)}{\left(\varphi_{n}\right)}\right] \otimes\left[\frac{\left(\kappa_{n}\right)}{\left(\mu_{n}\right)}\right] .
\end{aligned}
$$

This completes the proof.

\section{Conflict of Interests}

The authors declare that there is no conflict of interests regarding the publication of this paper.

\section{Acknowledgment}

The authors gratefully acknowledge that this research was partially supported by the University Putra Malaysia under the ERGS Grant Scheme having project no. 5527068.

\section{References}

[1] S. K. Q. Al-Omari and A. Kilıçman, "Unified treatment of the Krätzel transformation for generalized functions," Abstract and Applied Analysis, vol. 2013, Article ID 750524, 7 pages, 2013.

[2] E. Krätzel, "Eine Verallgemeinerung der Laplace- und MeijerTransformation," Wissenschaftliche Zeitschrift der FriedrichSchiller, vol. 14, pp. 369-381, 1965.

[3] D. I. Cruz-Báez and J. Rodríguez, “The $L_{\nu}^{(\rho)}$-transformation on McBride's spaces of generalized functions," Commentationes Mathematicae Universitatis Carolinae, vol. 39, no. 3, pp. 445452, 1998.

[4] G. L. N. Rao and L. Debnath, "A generalized Meijer transformation," International Journal of Mathematics and Mathematical Sciences, vol. 8, no. 2, pp. 359-365, 1985.

[5] A. H. Zemanian, Generalized Integral Transformations, Dover, New York, NY, USA, 2nd edition, 1987.

[6] V. Karunakaran and R. A. Chella Rajathi, "Gelfand transform for a Boehmian space of analytic functions," Annales Polonici Mathematici, vol. 101, no. 1, pp. 39-45, 2011.

[7] J. Beardsley and P. Mikusiński, "A sheaf of Boehmians," Annales Polonici Mathematici, vol. 107, no. 3, pp. 293-307, 2013.

[8] D. Nemzer, "One-parameter groups of Boehmians," Bulletin of the Korean Mathematical Society, vol. 44, no. 3, pp. 419-428, 2007.

[9] P. Mikusiński, "Fourier transform for integrable Boehmians," The Rocky Mountain Journal of Mathematics, vol. 17, no. 3, pp. 577-582, 1987.

[10] P. Mikusiński, “Tempered Boehmians and ultradistributions," Proceedings of the American Mathematical Society, vol. 123, no. 3, pp. 813-817, 1995.

[11] P. Mikusiński, “Convergence of Boehmians," Japanese Journal of Mathematics, vol. 9, no. 1, pp. 159-179, 1983. 
[12] S. K. Q. Al-Omari and A. Kılıçman, "On generalized HartleyHilbert and Fourier-Hilbert transforms," Advances in Difference Equations, vol. 2012, article 232, 12 pages, 2012.

[13] T. K. Boehme, "The support of Mikusiński operators," Transactions of the American Mathematical Society, vol. 176, pp. 319-334, 1973.

[14] D. Nemzer, "Boehmians on the torus," Bulletin of the Korean Mathematical Society, vol. 43, no. 4, pp. 831-839, 2006.

[15] V. Karunakaran and C. Ganesan, "Fourier transform on integrable Boehmians," Integral Transforms and Special Functions, vol. 20, no. 11-12, pp. 937-941, 2009.

[16] S. K. Q. Al-Omari and A. Kılıçman, "Some remarks on the extended Hartley-Hilbert and Fourier-Hilbert transforms of Boehmians," Abstract and Applied Analysis, vol. 2013, Article ID 348701, 6 pages, 2013.

[17] D. Nemzer, "A note on the convergence of a series in the space of Boehmians," Bulletin of Pure and Applied Mathematics, vol. 2, no. 1, pp. 63-69, 2008. 


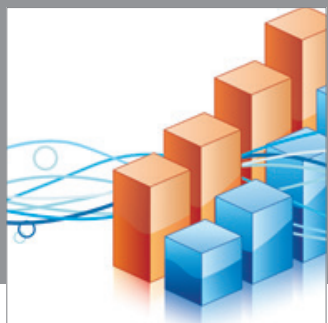

Advances in

Operations Research

mansans

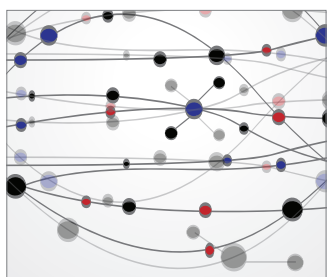

The Scientific World Journal
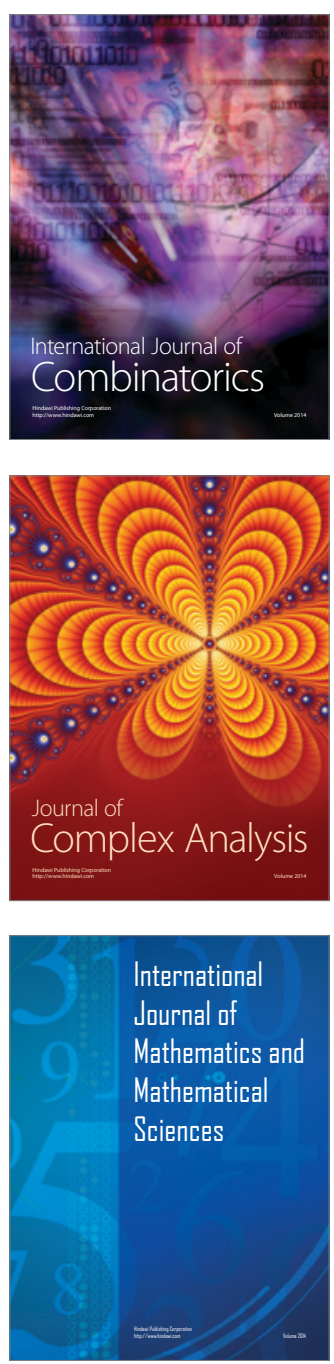
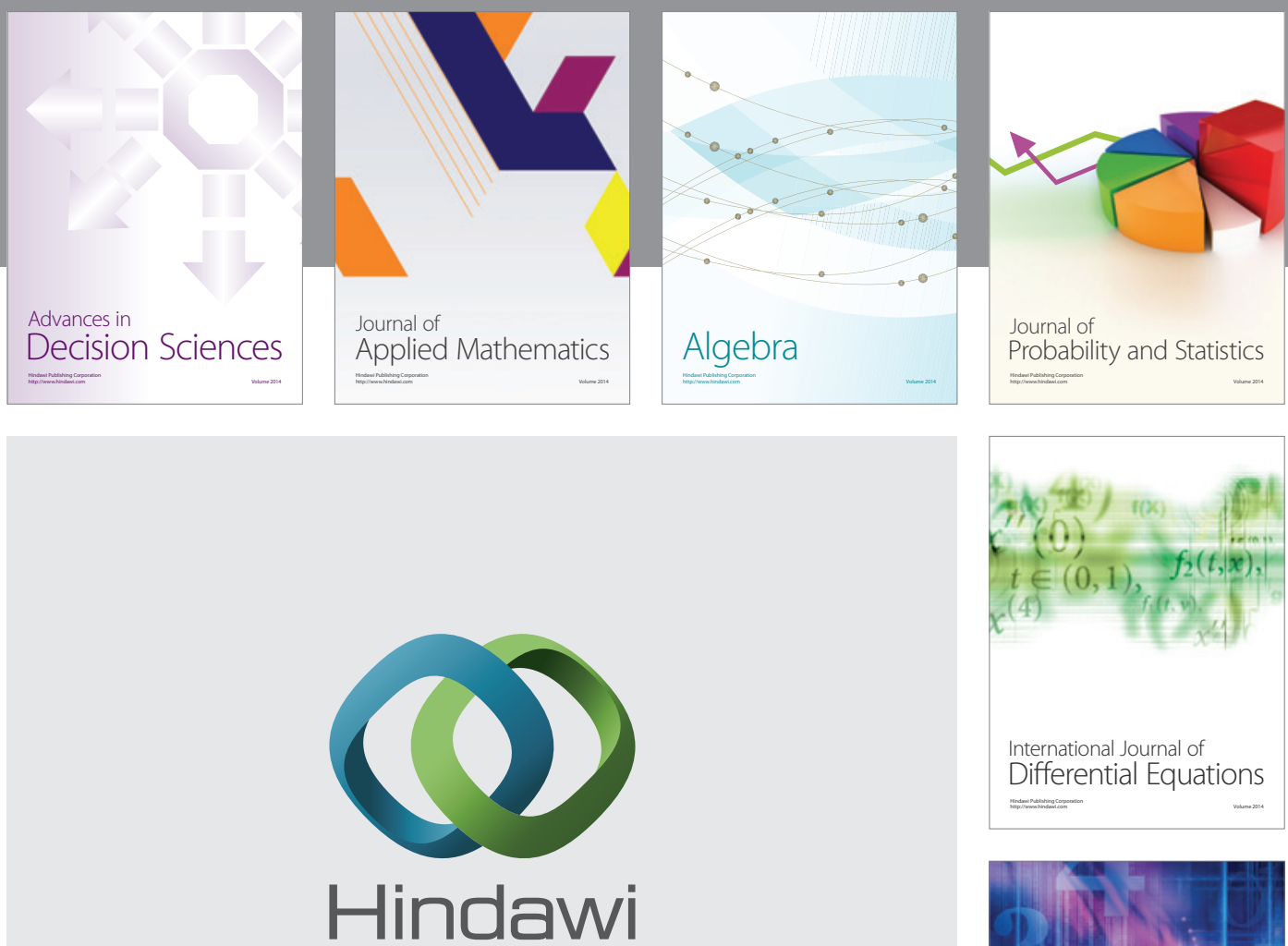

Submit your manuscripts at http://www.hindawi.com
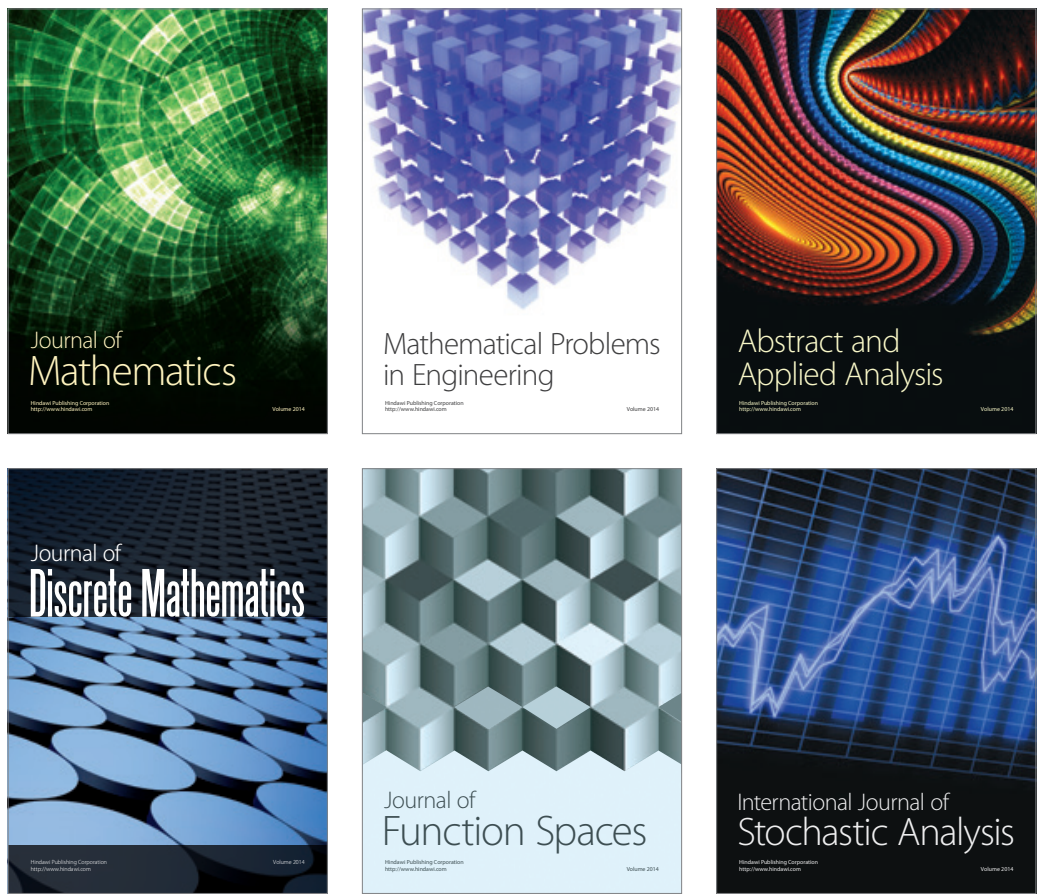

Journal of

Function Spaces

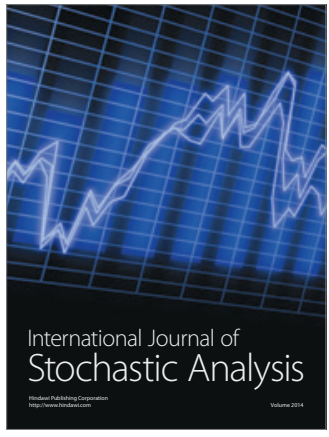

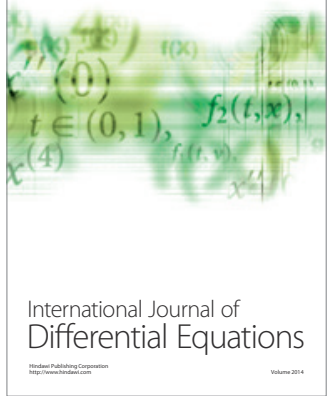
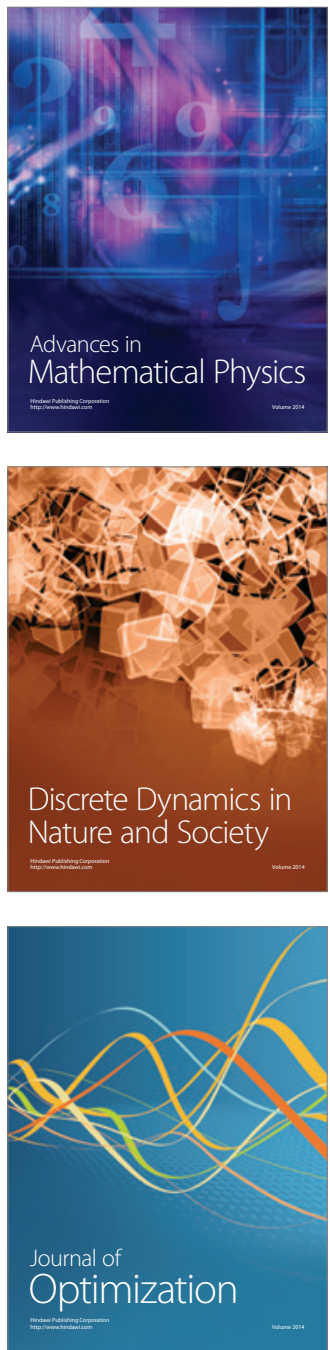